\title{
Suboptimal Disturbance Observer Design Using All Stabilizing $Q$ Filter for Precise Tracking Control
}

\author{
Wonhee Kim ${ }^{1}\left(\mathbb{D}\right.$ and Sangmin Suh ${ }^{2, *}$ (]) \\ 1 School of Energy Systems Engineering, Chung-Ang University, Seoul 06974, Korea; whkim79@cau.ac.kr \\ 2 Department of Information and Telecommunication Engineering, Gangneung-Wonju National University, \\ Wonju-si, Gangwon-do 26403, Korea \\ * Correspondence: sangminsuh@gwnu.ac.kr
}

Received: 20 July 2020; Accepted: 23 August 2020; Published: 26 August 2020

\begin{abstract}
For several decades, disturbance observers (DOs) have been widely utilized to enhance tracking performance by reducing external disturbances in different industrial applications. However, although a DO is a verified control structure, a conventional DO does not guarantee stability. This paper proposes a stability-guaranteed design method, while maintaining the DO structure. The proposed design method uses a linear matrix inequality (LMI)-based $H_{\infty}$ control because the LMI-based control guarantees the stability of closed loop systems. However, applying the DO design to the LMI framework is not trivial because there are two control targets, whereas the standard LMI stabilizes a single control target. In this study, the problem is first resolved by building a single fictitious model because the two models are serial and can be considered as a single model from the $\mathrm{Q}$-filter point of view. Using the proposed design framework, all-stabilizing $\mathrm{Q}$ filters are calculated. In addition, for the stability and robustness of the DO, two metrics are proposed to quantify the stability and robustness and combined into a single unified index to satisfy both metrics. Based on an application example, it is verified that the proposed method is effective, with a performance improvement of $10.8 \%$.
\end{abstract}

Keywords: disturbance observer (DO); $H_{\infty}$ control; linear matrix inequalities (LMIs); optimal control; Q filter; robustness; stability.

\section{Introduction}

Motion control systems are extensively used in different industrial applications such as hydraulic pumps, linear actuators, and rotating electric motors. In a control system, tracking performance is one of the main performance indicators because it implies the accuracy of the motion control system. Tracking error is defined by the position error signal (PES), that is, the difference between a reference command to be followed and the output of an actual plant, while tracking performance is measured by the deviation in the error of the measured tracking error. However, external disturbances act as unexpected commands and ultimately perturb the output of well-designed plants, resulting in a high PES. Therefore, external disturbances should be minimized. To eliminate external disturbances, a disturbance observer (DO) is widely utilized [1]. The DO is composed of disturbance estimation and a Q-filter to stabilize the DO. Because the DO uses a plant inverse model to estimate introduced disturbances, causality and instability problems are unavoidable. Furthermore, because the Q-filter can be trimmed only by controlling the bandwidth, it does not guarantee the stability of the DO. Causality and instability are fundamental challenges in a DO.

To reduce the external disturbances, numerous studies based on DOs have been undertaken; however, the majority of these studies have used DOs without considering stability. DOs have also been applied to satellite and star tracking systems [2]. In these systems, micro-electro-mechanical 
system (MEMS) accelerometers were used to measure the disturbances; however, the drift caused by the MEMS resulted in integral saturation. To remove this saturation, the study suggested a modified DO whereby a feedforward control was used instead of the feedback control in a conventional DO. However, the study had no stability analysis. For motor control systems, a practical design method to improve the stability and robustness of reaction-torque-observer-based force control systems was proposed [3]. In this study, to achieve robustness, high-frequency dynamics were attenuated by controlling the gains of the feedback loop. However, the method used was not a stability-guaranteed method; it only provided a guideline to address stability. To remove narrowband high-frequency disturbances, a discrete time DO based on an infinite impulse response filter was suggested and applied to a permanent-magnet synchronous motor [4]; however, stability was not guaranteed. Unlike a conventional DO, a high-pass Q-filter was used to estimate the disturbance for electro-hydraulic actuators [5]. The study analyzed only stability; it did not suggest a stability-guaranteed method. Two high-order DOs (HODOs) were also developed to estimate disturbances accurately for a pump-controlled hydraulic system [6]. The stability analysis was performed; however, the stability was not proved. DOs were applied to transportation because they also have motor-based actuators. A HODO was applied to mobile-wheeled inverted pendulum (MWIP) systems, and a HODO-based sliding mode control method was proposed to stabilize the underactuated MWIP systems and to reduce the chattering phenomenon [7]. In this study, robustness was evaluated by validating the bounded error dynamics to the bounded disturbances, and stability was achieved by the appropriate selection of the sliding surface coefficients. It was confirmed that DOs can be utilized to eliminate chatter problems rather than discontinuous control in antilock braking systems [8]; however, they did not investigate stability or robustness. In underactuated surface vehicles, a DO was used to address the actuator dead zones and disturbances [9]. The paper suggested only implicit stability and did not demonstrate robustness. To control the flight of an unmanned aerial vehicle, a DO was used to improve the tracking performance [10]. In this study, chirp disturbances were effectively estimated using a DO. The paper suggested a necessary condition for stability but did not guarantee this stability; robustness was not addressed at all. $H_{\infty}$-based DOs were introduced because the $H_{\infty}$ design method is sufficiently flexible to shape existing frequency responses with guaranteed stability [11,12]. A new complex coefficient adaptive DO based on back electromotive force estimation was designed using the $H_{\infty}$ design and was applied to the interior permanent-magnet synchronous motor drive [13]. This method was proven to be stable using a Lyapunov's direct method in the time domain. However, the proposed method was designed in the frequency domain because the introduced disturbances typically have complex frequency components, and to remove the composite disturbances the frequency domain design is more useful. In this study, a Q-filter was used as the complementary sensitivity of the DO because the real model was equivalent to the nominal model in the low-frequency range [14]. This study proved guaranteed stability in the frequency domain under the assumption of $P=P_{n}$, which is different from this study. For accurate mechanical manipulation, friction $[15,16]$ and uncertainty [17] were considered as disturbances. A DO was applied to remove the equivalent disturbances, and the stability in the above two studies was verified using the Lyapunov's direct method in the time domain. The Lyapunov's direct method is intuitive and capable of verifying the stability of linear systems and can be extended to nonlinear systems as well [18-21]. A stability-driven method for designing a DO in the frequency domain has rarely been attempted. Recently, an explicit Lyapunov-based proof for stability in the frequency domain has been suggested. Two switching DOs were used to obtain stability [22]. However, throughout the research, the assumption of $P=P_{n}$ was used, whereas the proposed method does not employ such an assumption. With this assumption, $Q$ and $1-Q$ were considered as sensitivity and complementary sensitivity functions, respectively, and the functions were shaped by weighting functions $W_{1}$ and $W_{2}$. Therefore, their design approach is different from the proposed method. In one of the other methods, a DO controller was first designed, and then a Q-filter was reconstructed indirectly in single-input single-output (SISO) [23] and multi-input multi-output (MIMO) [24] systems. In these studies, a two-input and one-output DO was designed, which is indeed 
a stability-driven DO. From the designed DO, $Q=-D_{1}$ and $P_{n}^{-1}=-D_{1}^{-1} D_{2}$ were reconstructed. However, the resulting controller using a linear matrix inequality (LMI)-based $H_{\infty}$ control is proper, that is, strictly proper or bi-proper (see Definition 1). Therefore, if $D_{1}$ is strictly proper, then $P_{n}^{-1}$ is not realizable, and additional filters are required to solve the causality problem. In another work on stability-driven DOs, an estimation-error-based DO in the state space was proposed [25]. In the study, the estimation errors are considered as equivalent disturbances because in the steady state, the estimation error is zero in the absence of disturbances. The method was realized in the state space to maintain the advantages of state-space controllers.

Because DOs are effective structures for eliminating disturbances and achieving high tracking performance, they have been used in numerous fields for several decades. However, thus far, no method has been proposed to ensure stability in the frequency domain while maintaining the structure of the existing DO. This study proposes a novel method to ensure continued stability of the $\mathrm{DO}$, while maintaining the structure of the existing DO. To stabilize the DO, an LMI-based $H_{\infty}$ control is used because the method can deliver control to always stabilize closed loop systems. However, applying the DO design to the LMI-based $H_{\infty}$ control framework is not trivial because the DO has two models, that is, a plant model and a nominal model, whereas a standard LMI-based $H_{\infty}$ control stabilizes only one model regardless of its type, that is, an SISO or MIMO model. To apply the DO design problem to an LMI framework, in this study, the plant and nominal models are considered as a single fictitious model because the two are serial and can be a single model from the control point of view in the DO. The proposed framework offers sufficient conditions for the DO stability, the resulting solution (Q-filter) is optimal for the weight functions and target model, and it always stabilizes the DO mathematically. However, although the Q-filter stabilizes the DO, for implementation, the DO should be represented in the form of $Q \cdot P_{n}^{-1} /(1-Q)$. Here, the fundamental problems of a DO-causality and instability-arise due to $P_{n}^{-1}$. A strictly proper $P_{n}$ makes a non-causal DO, and a non-minimum phase $P_{n}$ makes an unstable DO. In these cases, the DO cannot be realizable and/or is unstable. To solve the instability problem, this study proposes an all-filter-based inversion technique, which is a modified version of the previous study [26]. To resolve the causality problem, a new guideline for filter design is suggested. In the filter design guide, a phase distortion of the filter is underlined, and complex conjugated poles are recommended, which is definitely unique to this study. Secondly, metrics to increase the stability margins and decrease the high-frequency dynamics for robustness are proposed independently. Then, the two metrics are quantified independently and unified for considering both the stability and robustness simultaneously, which is also proposed, for the first time, in this paper. The contributions of this study are summarized as follows.

- The method provides a design framework for Q-filter design to always stabilize the DO for all types of models because the proposed method results from a Lyapunov-stability-driven $H_{\infty}$ control.

- To apply the DO design problem to the LMI-based control framework, both an identified and a nominal model are considered as the target model to be controlled, which is different from the design in conventional methods. Based on the idea that the Q-filter output should be equal to the introduced external disturbance in the frequency of interest, a new design framework for an LMI-based $H_{\infty}$ control is proposed, which is a new design concept.

- The proposed method provides easy controllability for both performance and robustness by simply adjusting the weight functions while maintaining stability.

- This study underlines the phase distortion of the compensator to solve the causality problem, and filters with complex conjugate poles are recommended.

- Unified metrics quantifying both the stability margin and high-frequency dynamics are suggested to realize a robustly stable DO.

The remainder of this paper is organized as follows. Section 2 contains the first result of the main research. In this section, a conventional DO is discussed. From the concept of the operation, a new 
framework for systematic Q-filter design is suggested, and the optimal Q-filter that stabilizes the DO is calculated. This study is the first attempt to obtain a stability-guaranteed method while maintaining the DO structure. Furthermore, this method offers easy controllability over stability and robustness. Section 3 discusses the second result. The transfer function of the DO is represented by a function of an inverse model of the nominal plant, which incurs instability and causality problems. To resolve the instability problem, an all-filter-based inverse model is proposed. To solve the causality problem, unified metrics via Nyquist plots are designed and proposed. To quantify and penalize the stability and robustness, a unified metric is suggested, which is introduced, for the first time, in this paper. In Section 4, illustrative examples including time and frequency responses are presented. To verify the precision tracking control, hard disk drives (HDDs) are used as a motion control system. Section 5 provides complete solutions according to the model types and limitations of the proposed method. The conclusion and future work follow.

\section{Stabilizing Q Filter Design}

A system with a DO has two control loops, the outer and inner loop systems. The outer loop system consists of a plant model and a controller that stabilizes the plant model. Because the controller of the outer loop system is designed in the absence of external disturbances, the outer loop system does not contain a DO, and the controlled plant output is the same as the estimate. In the presence of disturbances, the output cannot be estimated; therefore, a DO is added to minimize the disturbances. This DO forms an additional control loop system, that is, an inner loop system. Therefore, before designing a DO, an outer loop system must be designed. This section focuses on the DO design, and the outer loop design is described in Section 4.

\subsection{Conventional $D O$}

In the presence of external disturbances, the DO is designed to reduce the effect of the disturbances. A DO reduces the effect of the disturbances by subtracting the introduced disturbances. Therefore, the DO consists of a disturbance-monitoring filter and a stabilizing filter, that is, a Q-filter. In Figure 1, a conventional DO structure is depicted, where $u$ is the output of the state feedback controller $C$ that forms an outer loop control system.

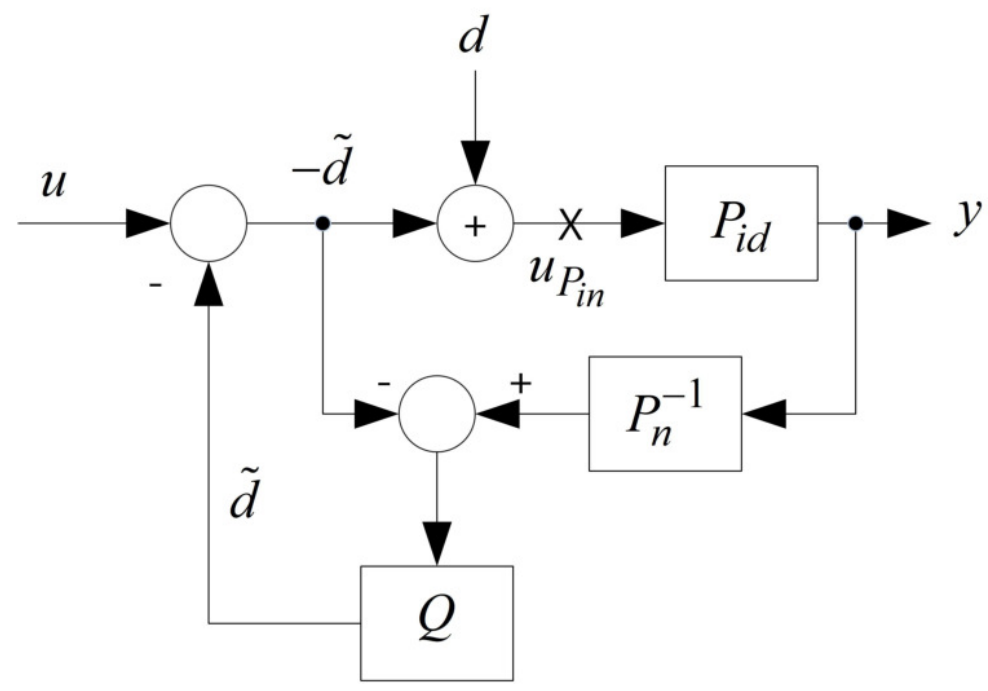

Figure 1. Conventional disturbance observer (DO) structure (inner loop system). 
Here, $P_{i d}$ and $P_{n}$ are an identified and nominal models respectively. The input of the Q-filter is written as

$$
\begin{aligned}
i_{Q} & =-u+P_{n}^{-1} \cdot P_{i d} \cdot(d+u) \\
& =-u+P_{n}^{-1} \cdot P_{i d} \cdot d+P_{n}^{-1} \cdot P_{i d} \cdot u
\end{aligned}
$$

If $P_{i d}$ and $P_{n}$ are ideally identical, $i_{Q}$ equals $d$ because $P_{n}^{-1} \cdot P_{i d}=1$. The DO must be a systematic method to reduce the disturbance; however, the stabilization Q-filter is generally designed by the trial and error method because the Q-filter is a typical low-pass filter with zero DC gain. Therefore, the controlling factor is only a bandwidth or could be a skirt feature (transition band) of the filter.

\subsection{Proposed Framework for Optimal Stabilizing Q Filter Design}

In this subsection, a novel stabilizing $\mathrm{Q}$ filter design is proposed. In an ideal $\mathrm{DO}, \tilde{d}$ should be equal to $d$ in the frequency range of interest, which implies that the input of $P_{i d}, u_{P_{i n}}$, should be minimized in the corresponding frequency range. Moreover, because the DO has inverse dynamics, and high-frequency dynamics can be easily excited, it is desirable to weight the control effort of the DO. In addition, from the Q-filter point of view, a plant to be controlled is $P=P_{i d} \cdot P_{n}^{-1}$ because $P_{i d}$ and $P_{n}^{-1}$ successively exist.

For these reasons, an augmented and generalized system is proposed, as displayed in Figure 2. Using the block diagram, an optimal $Q$ filter based on $H_{\infty}$ control is designed. For the weighting function design, the following function is used:

$$
W(s)=\frac{\left(s / M^{1 / n}+\omega_{B}^{*}\right)^{n}}{\left.\left(s+\omega_{B}^{*} A^{1 / n}\right)\right)^{n}}
$$

where $M, A, \omega_{B}^{*}$ and $n$ are the upper bound, lower bound, bandwidth, and order of $\left|W^{-1}(s)\right|$ respectively [27]. In the proposed framework, $W_{1}^{-1}$ controls the ability of the disturbance rejection, and $\mathrm{W}_{2}^{-1}$ controls the robustness. The detailed discussion is presented in Section 4.

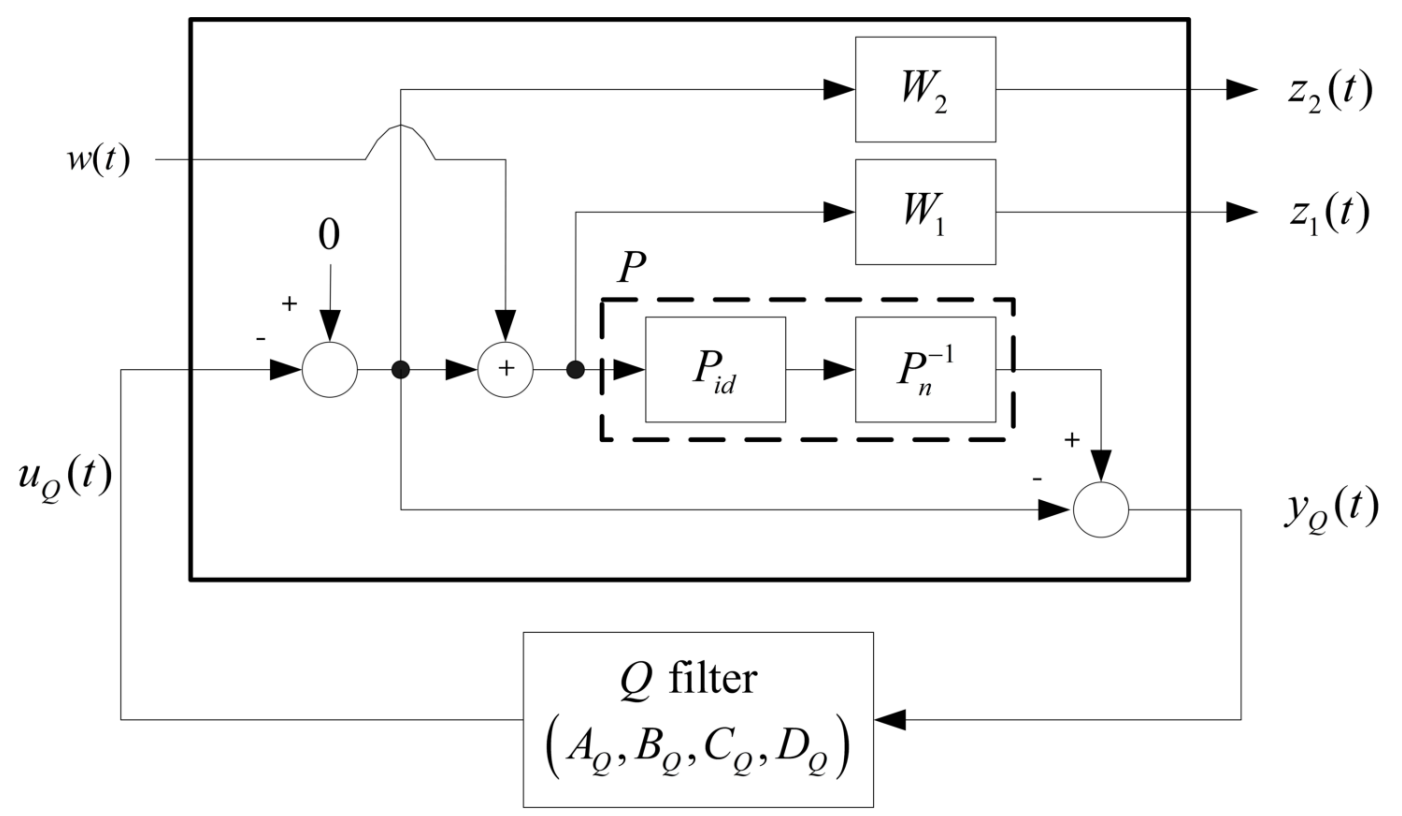

Figure 2. Framework for proposed DO design. 


\subsection{Optimal Stabilizing Q Filter Design}

To realize the framework as displayed in Figure 2, all transfer functions must be described using state-space representation. Here, $P=P_{i d} \cdot P_{n}^{-1}$ is proper because $P=P_{r}$, and $P_{r}$ is proper. Thus, $P$ is realizable and can be written using state-space representation.

$$
P: \begin{array}{r}
\dot{x_{p}}(t)=A_{p} x_{p}(t)+B_{p} u_{p}(t) \\
y_{p}(k)=C_{p} x_{p}(t)+D_{p} u_{p}(t)
\end{array}
$$

Here, $W_{1}$ for disturbance rejection performance and $W_{2}$ for controlling uncertainty are also described using state-space representation, as shown in (4) and (5).

$$
\begin{aligned}
& W_{1}: \dot{x}_{W_{1}}(t)=A_{W_{1}} x_{W_{1}}(t)+B_{W_{1}} u_{W_{1}}(t) \\
& W_{2}: \begin{array}{l}
x_{W_{2}}(t)=A_{W_{2}} x_{W_{2}}(t)+B_{W_{2}} u_{W_{2}}(t) \\
y_{W_{2}}(k)=C_{W_{2}} x_{W_{2}}(t)+D_{W_{2}} u_{W_{2}}(t)
\end{array}
\end{aligned}
$$

Using (3), the plant state equation can be represented by

$$
\begin{aligned}
\dot{x}_{p}(t) & =A_{p} x_{p}(t)+B_{p}\left(w(t)-u_{Q}(t)\right) \\
& =A_{p} x_{p}(t)+B_{p} w(t)-B_{p} u_{Q}(t) .
\end{aligned}
$$

With (4) and (5), the corresponding state equations are captured by

$$
\begin{gathered}
\dot{x}_{W_{1}}(t)=A_{W_{1}} x_{W_{1}}(t)+B_{W_{1}}\left(w(t)-u_{Q}(t)\right) \\
=A_{W_{1}} x_{W_{1}}(t)+B_{W_{1}} w(t)-B_{W_{1}} u_{Q}(t), \\
\dot{x}_{W_{2}}(t)=A_{W_{2}} x_{W_{2}}(t)+B_{W_{2}}\left(-u_{Q}(t)\right) \\
=A_{W_{2}} x_{W_{2}}(t)-B_{W_{2}} u_{Q}(t) .
\end{gathered}
$$

The output to be minimized are $z_{1}(t)$ and $z_{2}(t)$ and are calculated by

$$
\begin{gathered}
z_{1}(t)=C_{W_{1}} x_{W_{1}}(t)+D_{W_{1}}\left(w(t)-u_{Q}(t)\right) \\
=C_{W_{1}} x_{W_{1}}(t)+D_{W_{1}} w(t)-D_{W_{1}} u_{Q}(t), \\
z_{2}(t)=C_{W_{2}} x_{W_{2}}(t)+D_{W_{2}}\left(-u_{Q}(t)\right) \\
=C_{W_{2}} x_{W_{2}}(t)-D_{W_{2}} u_{Q}(t) .
\end{gathered}
$$

The input of the Q-filter is also written as

$$
\begin{aligned}
y(t) & =C_{p} x_{p}(t)+D_{p}\left(w(t)-u_{Q}(t)\right)+u_{Q}(t) \\
& =C_{p} x_{p}(t)+D_{p} w(t)+\left(1-D_{p}\right) u_{Q}(t) .
\end{aligned}
$$

Using (6)-(11), the augmented systems can be written as

$$
\begin{aligned}
\dot{x}(t) & =A x(t)+B_{1} w(t)+B_{2} u_{Q}(t) \\
z(t) & =C_{1} x(t)+D_{11} w(t)+D_{12} u_{Q}(t) \\
y_{Q}(t) & =C_{2} x(t)+D_{21} w(t)+D_{22} u_{Q}(t),
\end{aligned}
$$


where $x(t)=\left[\begin{array}{lll}x_{p}^{T}(t) & x_{W 1}^{T}(t) & x_{W 2}^{T}(t)\end{array}\right]^{T}, z(t)=\left[\begin{array}{ll}z_{1}^{T}(t) & z_{2}^{T}(t)\end{array}\right]^{T}$ and

$$
\begin{gathered}
A=\left[\begin{array}{ccc}
A_{p} & 0 & 0 \\
0 & A_{W_{1}} & 0 \\
0 & 0 & A_{W_{2}}
\end{array}\right], B_{1}=\left[\begin{array}{c}
B_{p} \\
B_{W_{1}} \\
0
\end{array}\right], B_{2}=\left[\begin{array}{c}
-B_{p} \\
-B_{W_{1}} \\
-B_{W_{2}}
\end{array}\right], \\
C_{1}=\left[\begin{array}{ccc}
0 & C_{W_{1}} & 0 \\
0 & 0 & C_{C_{W_{2}}}
\end{array}\right], D_{11}=\left[\begin{array}{c}
D_{W_{1}} \\
0
\end{array}\right], D_{12}=\left[\begin{array}{l}
-D_{W_{1}} \\
-D_{W_{2}}
\end{array}\right], \\
C_{2}=\left[\begin{array}{lll}
C_{p} & 0 & 0
\end{array}\right], D_{21}=\left[D_{p}\right], D_{22}=\left[1-D_{p}\right] .
\end{gathered}
$$

Using (12), the stabilizing Q-filter is calculated as follows.

$$
Q: \begin{aligned}
& x_{Q}(t)=A_{Q} x_{Q}(t)+B_{Q} y_{Q}(t) \\
& u_{Q}(k)=C_{Q} x_{Q}(t)+D_{Q} y_{Q}(t) .
\end{aligned}
$$

Theorem 1. Q-filter stabilizes $P_{i d} \cdot P_{n}^{-1}$.

Proof of Theorem 1. Since $Q$ is a Lyapunov-stability-based $H_{\infty}$ dynamic output feedback controller, $\mathrm{Q}$ always stabilizes a fictitious plant, $P_{i d} \cdot P_{n}^{-1}[28-30]$.

\section{DO Realization}

Even though an optimal Q-filter that stabilize the inner loop was designed in the previous subsection, a complete DO must be designed considering $P_{n}^{-1}$ as in Figure 1 . To realize $P_{n}^{-1}$, we could encounter two problems, causality and instability. Causality is related to the difference between the orders of the numerator and denominator. Let the transfer function $T$ be given by $N_{T} / D_{T}$, where $N_{T}$ and $D_{T}$ are a numerator and a denominator of $T$. Then, $d=\rho\left(D_{T}\right)-\rho\left(N_{T}\right)$ is referred to as relative degree, where $\rho(\cdot)$ denotes the order of the corresponding polynomial. According to the relative degree, $T$ can be categorized as follows [27].

\section{Definition 1.}

- If $d>0, T$ is strictly proper and can be realizable.

- If $d=0, T$ is bi-proper and can be realizable.

- If $d<0, T$ is improper and cannot be realizable because of causality.

If $P_{n}$ is a non-minimum phase plant with unstable zeros, then instability arises. Therefore, to realize DO, the two problems, causality and instability, must be resolved. From Figure 1, DO can be written as

$$
C_{D O}^{0}=\left.\frac{-\tilde{d}}{y}\right|_{u=0}=\frac{Q \cdot P_{n}^{-1}}{1-Q}
$$

To realize $C_{D O}^{0}, P_{n}^{-1}$ must be calculated. However, $P_{n}$ could be a non-minimum phase or a strictly proper function. In this case, $C_{D O}^{0}$ becomes unstable and unrealizable. 


\subsection{Solution to Non-Minimum Phase Plants}

To solve the instability problem, an alternative inverse model based on an all-pass filter is used. In previous research [26], discrete time-domain all-pass filters were introduced. In this study, continuous time-domain all-pass filters are developed. Let $P_{n}$ be given by

$$
P_{n}=\frac{B_{s}(s) B_{u s}(s)}{A(s)},
$$

where $B_{s}(s)$ and $B_{u s}(s)$ are the numerators containing stable and unstable zeros, respectively. $A(s)$ is a polynomial of the denominator. Then, rather than $P_{n}^{-1}$, we can use

$$
\bar{P}_{n}^{-1}=\frac{A(s)}{B_{s}(s) \bar{B}(s)} .
$$

Furthermore, $\bar{B}(s)$ is obtained by

$$
\frac{B_{u s}(s)}{\bar{B}(s)}=1 \angle \phi,
$$

where the roots of $\bar{B}(s)$ are mirrored images of the roots of $B_{u s}(s)$. Because the gain is unity, $B_{u s}(s)$ can be substituted with $\bar{B}(s)$ to calculate the inverse model. Thus, the DO is rewritten as

$$
C_{D O}^{1}=\frac{Q}{1-Q} \cdot \frac{A(s)}{B_{s}(s) \bar{B}(s)}
$$

\subsection{Solution to Strictly Proper Plants}

The resulting $C_{D O}^{1}$ could be improper because $P_{n}$ and $\bar{P}_{n}$ could be strictly proper. To solve this causality problem, additional filters must be designed. In this report, a compensation-filter design method is proposed that considers stability and robustness. The additional compensator is defined by

$$
C_{\text {comp }}^{0}=\prod_{i=1}^{d} \frac{1}{s / \tau_{i}+1},
$$

where $d$ denotes the relative degree of $P_{n}$, and $\tau_{i}$ is a pole of the compensator to be designed.

Proposition 1. If $\tau_{i}$ is far from the origin in the left-half plane, the DO is more stable.

The additional $C_{\text {comp }}$ inevitably decreases the stability margins because of the time delay caused by the phase distortion of the poles. Accordingly, if the pole is designed to be located far from origin, then the DO is more stable.

Proposition 2. A second-order filter with two complex conjugate poles decreases the stability margins less than a second-order filter with two real poles because the former has a faster phase transient, which means a smaller phase loss near the gain crossover frequency of the open-loop transfer function.

Therefore, the use of a second-order filter with two complex conjugate poles is recommended for DO stability, which is represented as

$$
C_{\text {comp }}^{1}=\prod_{i=1}^{q} \frac{1}{s / \tau_{i}+1} \cdot \prod_{j=1}^{p} \frac{\tau_{j}^{2}}{s^{2}+2 \zeta_{j} \tau_{j} s+\tau_{j}^{2}},
$$


where $p$ and $q$ are integers satisfying $d=2 \times p+q$. Then DO is calculated by

$$
C_{D O}=\frac{Q}{1-Q} \cdot \frac{A(s)}{B_{s}(s) \bar{B}(s)} \cdot \prod_{i=1}^{q} \frac{1}{s / \tau_{i}+1} \cdot \prod_{j=1}^{p} \frac{\tau_{j}^{2}}{s^{2}+2 \zeta_{j} \tau_{j} s+\tau_{j}^{2}} .
$$

Moreover, high-frequency dynamics must be considered. If high-frequency dynamics exist over the phase crossover frequency, the high frequency uncertainty could be excited regardless of the stability margins.

\subsection{General Solution with Considering Stability and Robustness}

A typical Nyquist plot of the open-loop transfer function with high-frequency dynamics is illustrated in Figure 3.

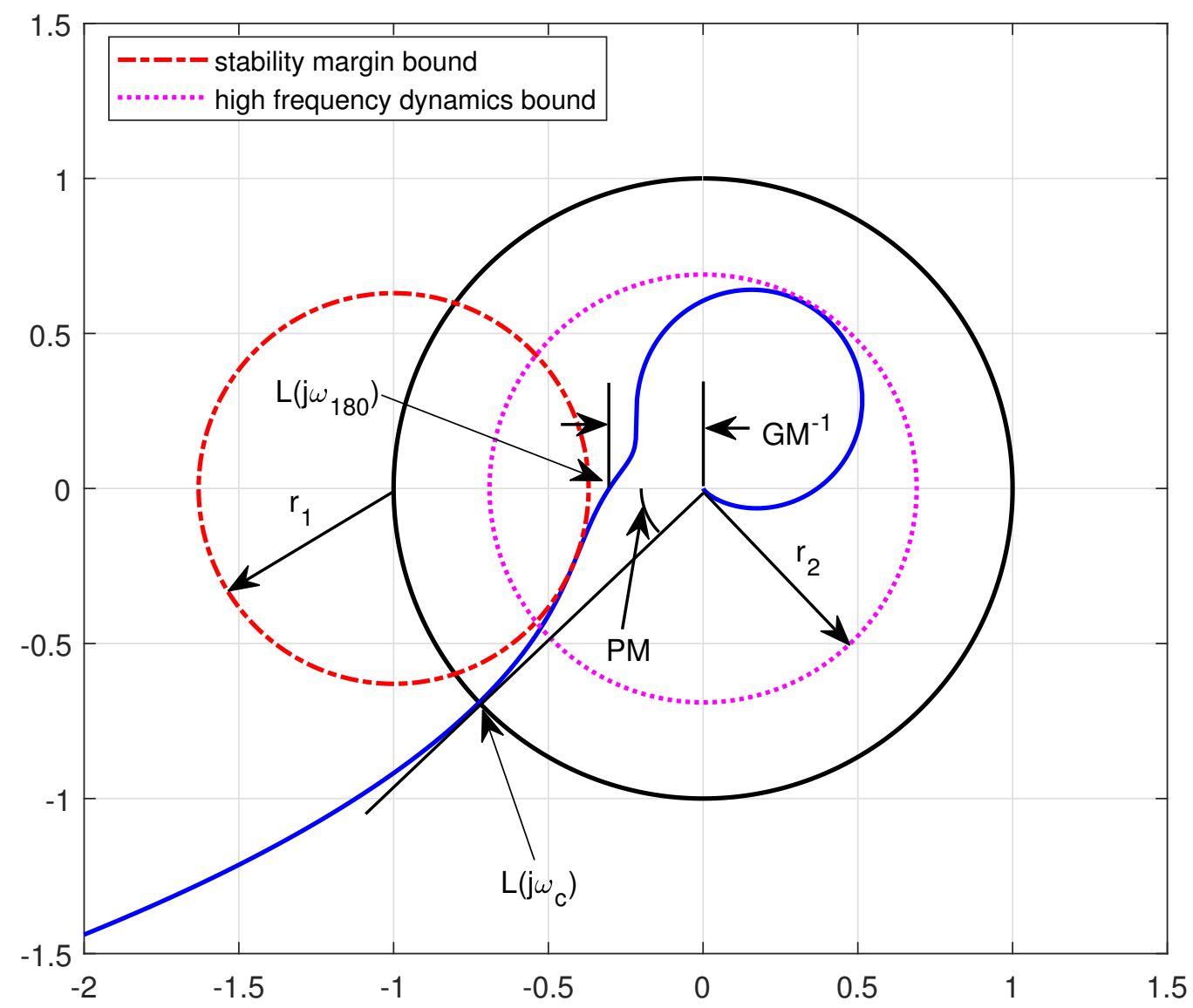

Figure 3. Nyquist plot with stability margin and high frequency dynamics.

Here, $\omega_{c}$ and $\omega_{180}$ are the gain and phase crossover frequencies of the open-loop transfer function, $L(j \omega)$, respectively. In addition, GM and PM are the gain and phase margins, respectively. Therefore, for sufficient stability margin, $\left|L\left(j \omega_{180}\right)\right|$ should be small, and $L L\left(j \omega_{c}\right)-180$ should be large, which means $r_{1}$ of the circle tangent to $L(j \omega)$ should be as large as possible. To reduce the high-frequency dynamics over the phase crossover frequencies, $r_{2}$ of the circle tangent to $L(j \omega)$ should be as small as possible. The first circle is located at $(-1,0)$; the second is located at $(0,0)$. To combine two metrics into one metric, a new parameter $\alpha$ is introduced, and the unified metric can be written as

$$
M=\alpha r_{1}+(1-\alpha) r_{2}^{-1}
$$


where $\alpha$ is a weight factor for controlling stability margin and high-frequency dynamics excitation, and $0 \leq \alpha \leq 1$. Therefore, for maximum stability margin and minimum excitation of the high-frequency dynamics, $C_{\text {comp }}$ must be designed to maximize $M$. Accordingly, the DO maximizing $M$ can be obtained as

$$
C_{D O}^{*}=\frac{Q}{1-Q} \cdot \frac{A(s)}{B_{s}(s) \bar{B}(s)} \cdot \prod_{i=1}^{q} \frac{1}{s / \tau_{i}^{*}+1} \cdot \prod_{j=1}^{p} \frac{\left(\tau_{j}^{*}\right)^{2}}{s^{2}+2 \zeta_{j}^{*} \tau_{j}^{*} s+\left(\tau_{j}^{*}\right)^{2}}
$$

where $*$ denotes the optimal values to maximize $M$. Equation (23) is a general solution for all types of models; each solution based on a model type is presented in Section 5.

\section{Application Example}

This section presents the modeling, the outer loop design based on state feedback controller using a nominal plant $P_{n}$, and the inner loop design based on the proposed method using the nominal plant $P_{n}$ and an identified model $P_{i d}$. In addition, the designed time and frequency responses are presented.

The proposed design method was applied to HDDs, as displayed in Figure 4, which are typical motion control systems. The dynamics of the plant model were measured using a laser Doppler vibrometer (LDV) and a dynamic signal analyzer (DSA). From the data, an identified model $P_{i d}$ with high frequency dynamics was captured by

$$
\begin{aligned}
P_{i d}(s) & =\frac{K_{t}}{J_{m}} \cdot \frac{1}{s^{2}+\left(B_{d} / J_{m}\right) s+K_{s} / J_{m}} \cdot \frac{\omega_{n}^{2}}{s^{2}+2 \zeta_{n} \omega_{n} s+\omega_{n}^{2}} \\
& =P_{n}(s) \cdot P_{r}(s),
\end{aligned}
$$

where $P_{n}(s)$ and $P_{r}(s)$ are the nominal and resonance models, respectively, and are defined as

$$
\begin{aligned}
& P_{n}(s)=\frac{K_{t}}{J_{m}} \cdot \frac{1}{s^{2}+\left(B_{d} / J_{m}\right) s+K_{s} / J_{m}}, \\
& P_{r}(s)=\frac{\omega_{n}^{2}}{s^{2}+2 \zeta_{n} \omega_{n} s+\omega_{n}^{2}},
\end{aligned}
$$

where $K_{t}$ is the torque constant, $J_{m}$ is the inertia, $B_{d}$ is the damping coefficient, and $K_{s}$ is the spring coefficient, which determines the low-frequency poles. Here, $\omega_{n}$ and $\zeta_{n}$ describe high-frequency resonance characteristics. The values of the parameters are listed in Table 1.

Table 1. HDDs parameters.

\begin{tabular}{llll}
\hline Parameter & Symbol & Value & Unit \\
\hline torque constant & $K_{t}$ & 0.053356 & $\mathrm{~N} \cdot \mathrm{m} / \mathrm{A}$ \\
inertia & $J_{m}$ & $30.34 \times 10^{-7}$ & $\mathrm{~kg} \cdot \mathrm{m}^{2}$ \\
damping coefficient & $B_{d}$ & 0.0013344 & $\mathrm{~N} \cdot \mathrm{s} / \mathrm{m}$ \\
spring coefficient & $K_{s}$ & 0.58691 & $\mathrm{~N} / \mathrm{m}$ \\
resonance frequency & $\omega_{n}$ & $2 \pi 5930$ & radian \\
damping ratio & $\zeta_{n}$ & 0.04 & - \\
\hline
\end{tabular}

The frequency responses of $P_{n}$ and $P_{i d}$ are illustrated in Figure 4. 

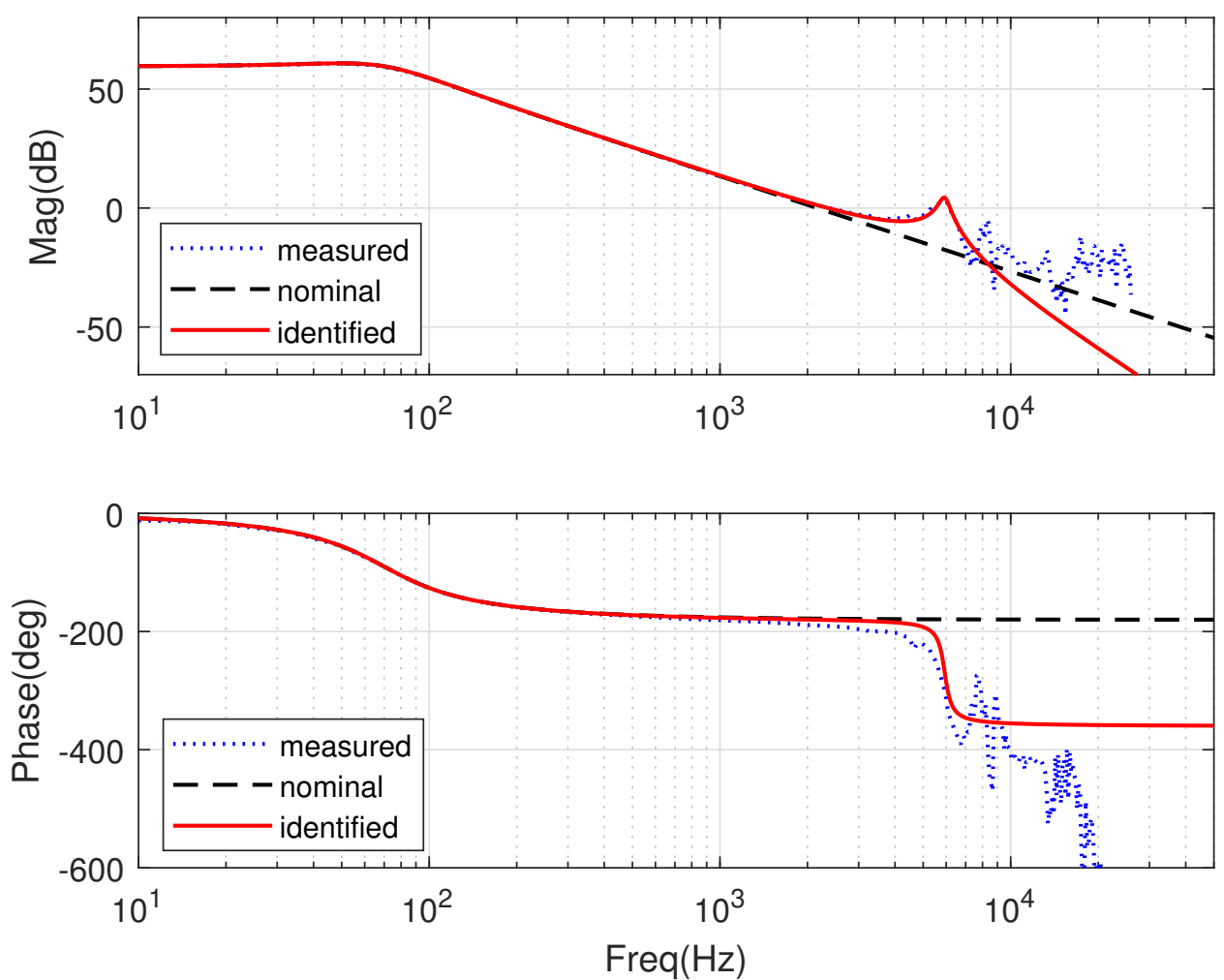

Figure 4. Frequency responses of measured, identified, and nominal models.

In this study, a state-estimator-based state-feedback controller was used to stabilize outer loop systems [31]. For the outer loop design, a nominal model $P_{n}$ based controller is designed. For a given plant $P_{n}$, represented by state space representation of

$$
P_{n}: \begin{array}{r}
\dot{x}_{n}(t)=A_{n} x(t)+B_{n} u(t) \\
y_{n}(t)=C_{n} x(t)+D_{n} u(t),
\end{array}
$$

an estimator-based state feedback controller was designed as follows.

$$
\begin{aligned}
\dot{\bar{x}}(t) & =A_{n} \bar{x}(t)+B_{n} u(t)+L\left(y_{n}(t)-C_{n} \bar{x}(t)\right) \\
C: \quad & =\left(A_{n}-B_{n} K-L C_{n}\right) \bar{x}(t)+L y_{n}(t) \\
u(t) & =-K \bar{x}(t)
\end{aligned}
$$

In (28), $L$ and $K$ are the estimator and controller gains, respectively. The designed open-loop transfer function of the outer loop is depicted in Figure 5.

The designed cutoff frequency of the open-loop function of the outer loop is $960 \mathrm{~Hz}$ to obtain reasonable stability margins, i.e., gain margin (GM) and phase margin (PM).

To design inner loop systems (DO), a stabilizing Q-filter should be designed. To design the proposed Q-filter, weighting functions should be designed. Equation (2) was used for weighting functions design; their frequency responses are displayed in Figure 6. 

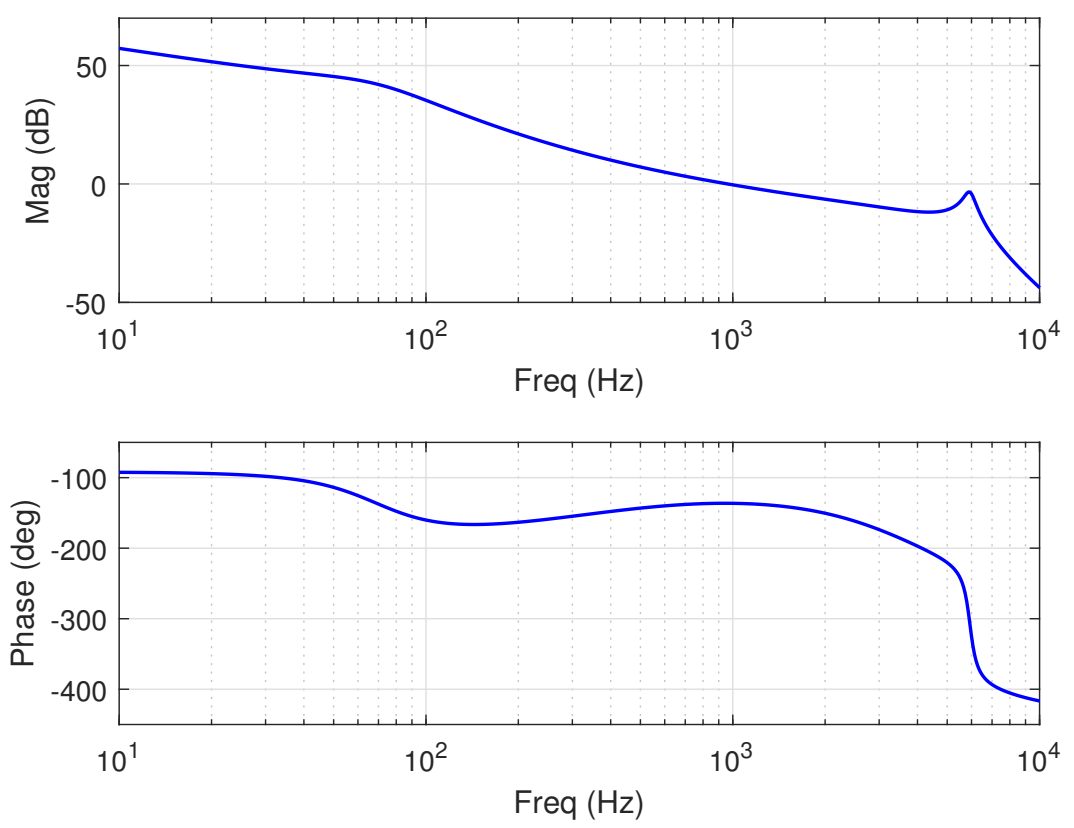

Figure 5. Open loop transfer function $\left(P_{i d} \cdot C\right)$.

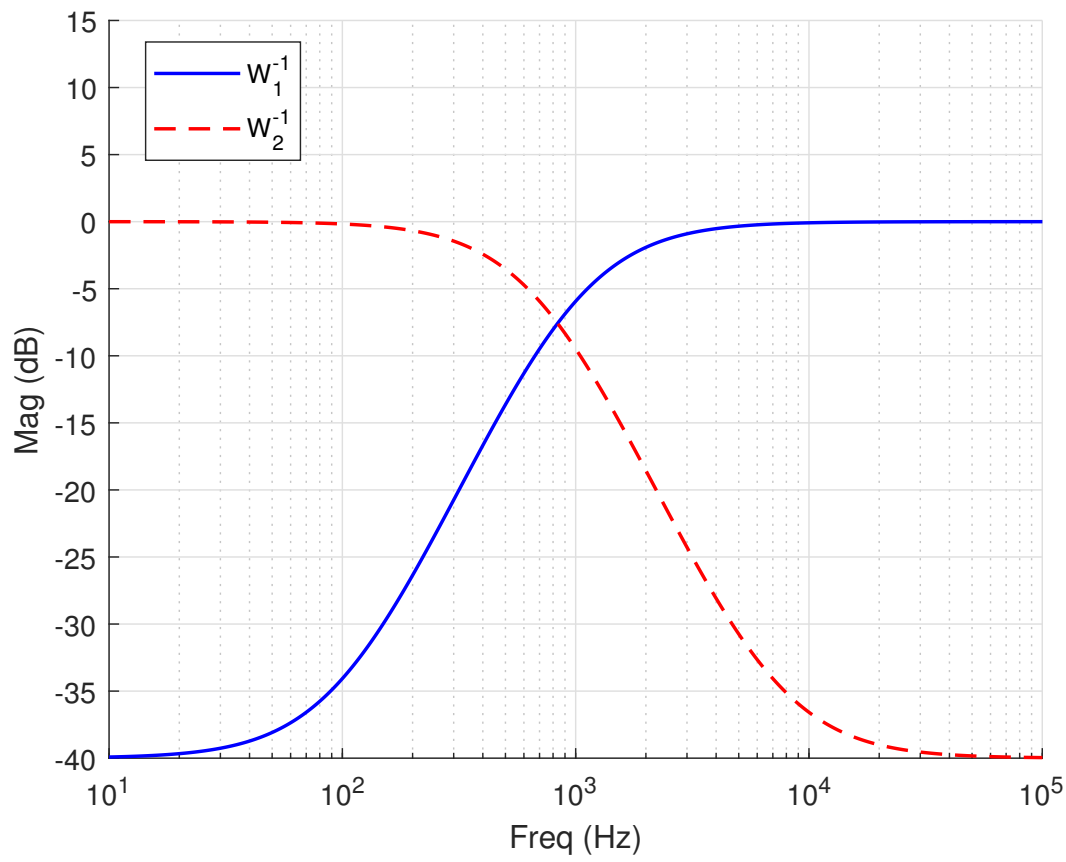

Figure 6. Frequency responses of $W_{1}^{-1}$ and $W_{2}^{-1}$.

Here, $W_{1}^{-1}$ determines the ability of the disturbance rejection. As the bandwidth of $W_{1}^{-1}$ increases, disturbances in a wider frequency range can be reduced. As the depth of $W_{1}^{-1}$ increases, the disturbances rejection performance increases. In Figure 6, the cutoff frequency of $W_{1}^{-1}$ is $1 \mathrm{KHz}$, which implies that $W_{1}^{-1}$ can reduce disturbances located in lower frequency of $1 \mathrm{KHz}$. The gain of $W_{1}^{-1}$ is $-40 \mathrm{~dB}$ at $10 \mathrm{~Hz}$, which implies that when an external disturbance (d) with $10 \mathrm{~Hz}$ frequency component is introduced, the designed $Q$ filter generates $\tilde{d}$ that satisfies $|d-\tilde{d}|=10^{(-40 / 20)}=0.01$. This means that $\tilde{d} \approx d$ and $\tilde{d}$ subtracts $d$ from the total control effort. Therefore, the effects of $d$ are reduced on the basis of (1). The error $|d-\tilde{d}|$ can be further minimized by reducing $A$ of $W_{1}$. However, an excessive control effort would be designed, and this is not recommended. $W_{2}^{-1}$ represents robustness because it can reduce the excitation of the high-frequency dynamics. In Figure 6, the cutoff 
frequency is approximately $500 \mathrm{~Hz}$, which means that uncertainties located in above $500 \mathrm{~Hz}$ can be reduced. In the design example, the gain of $W_{2}^{-1}$ is $-40 \mathrm{~dB}$ at $100 \mathrm{KHz}$. Let $\Phi$ be a transfer function from $w(t)$ to the input of $W_{2}$, then we obtain $\left|z_{2} / w\right|=\left|\Phi \cdot W_{2}\right|=\gamma$, where $\gamma$ is positive and a constant value to be minimized in the LMI framework. The equation can also be represented by $|\Phi|=\gamma\left|W_{2}^{-1}\right|$, which means that the frequency response of $\Phi$ is less than the scaled $\left|W_{2}^{-1}\right|$ in all frequency range. In general, $\gamma=1$. This also implies that the gain of $\Phi$ (transfer function from $w$ to the input of $W_{2}$ ) is $-40 \mathrm{~dB}$ at $100 \mathrm{KHz}$, resulting in the suppression of the high-frequency dynamics. In this study, second-order weighting functions were designed, that is, $n=2$. The other parameters are summarized in Table 2.

Table 2. Parameters of $W_{1}$ and $W_{2}$.

\begin{tabular}{llll}
\hline Symbol & $\boldsymbol{W}_{\mathbf{1}}$ & $\boldsymbol{W}_{\mathbf{2}}$ & Unit \\
\hline $\mathrm{M}$ & 0 & -40 & $\mathrm{~dB}$ \\
$\mathrm{~A}$ & -40 & 0 & $\mathrm{~dB}$ \\
$\omega_{B}^{*}$ & 1000 & 7000 & $\mathrm{~Hz}$ \\
\hline
\end{tabular}

With the above weighting functions, a stabilizing $Q$ filter was designed. Equations (16) and (20) were used to design the inverse model and compensator. In this work, $P_{n}$ is a minimum phase plant with relative degree of 2 . Thus, a second-order filter was used as a compensator, and $\left.M\right|_{\alpha=0.5}$ was used as a single criterion to design $C_{D O}^{*}$ for a fair penalty on both stability and robustness. The designed open-loop transfer functions are illustrated in Figure 7. The DO forming the inner loop increases the loop gain of the total open-loop transfer function, which is controlled by the cutoff frequency of $W_{1}^{-1}$. In addition, the high-frequency dynamics are controlled by cutoff frequency of $\mathrm{W}_{2}^{-1}$.

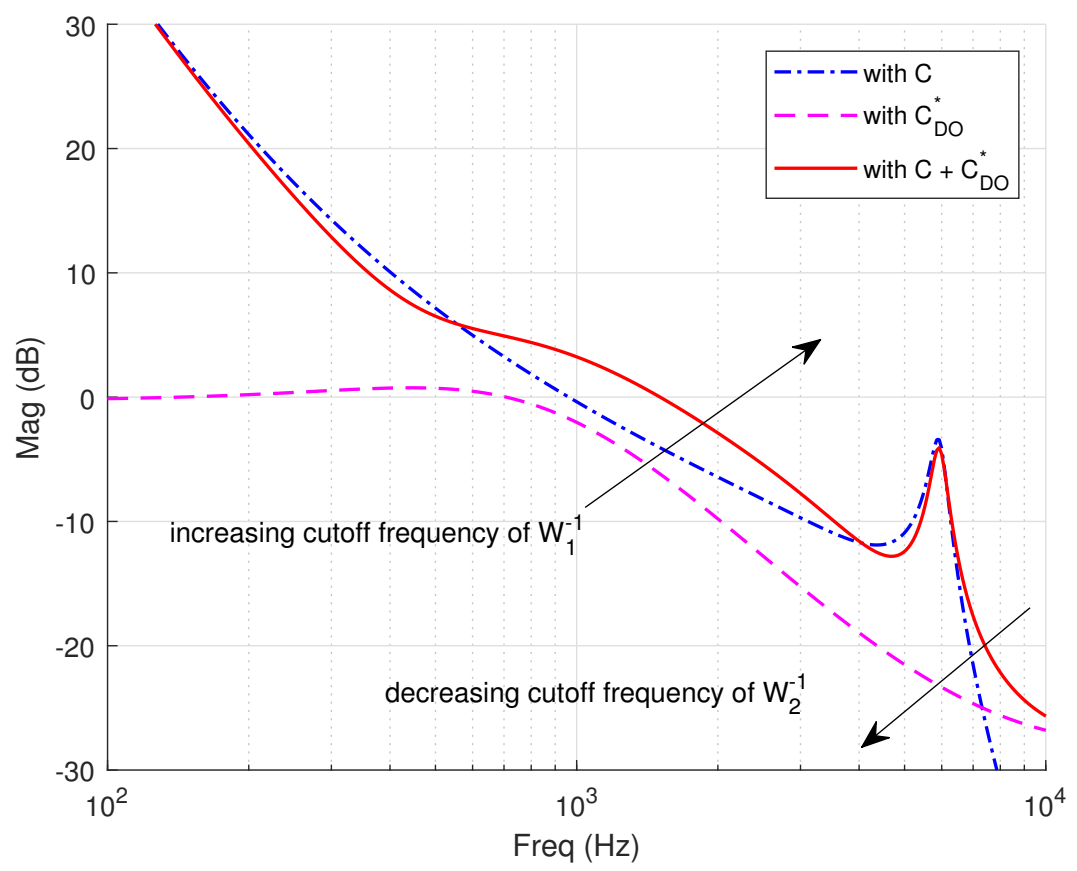

Figure 7. Designed open loop transfer functions.

The designed sensitivity functions are compared in Figure 8. Because the sensitivity function is defined by a transfer function from $d$ to $u_{P_{i n}}$ as displayed in Figure 1, this function indicates how similar $-\tilde{d}$ is to $d$. The proposed DO improved the performance of the disturbance rejection in the frequency range from $500 \mathrm{~Hz}$ to $3 \mathrm{kHz}$. 


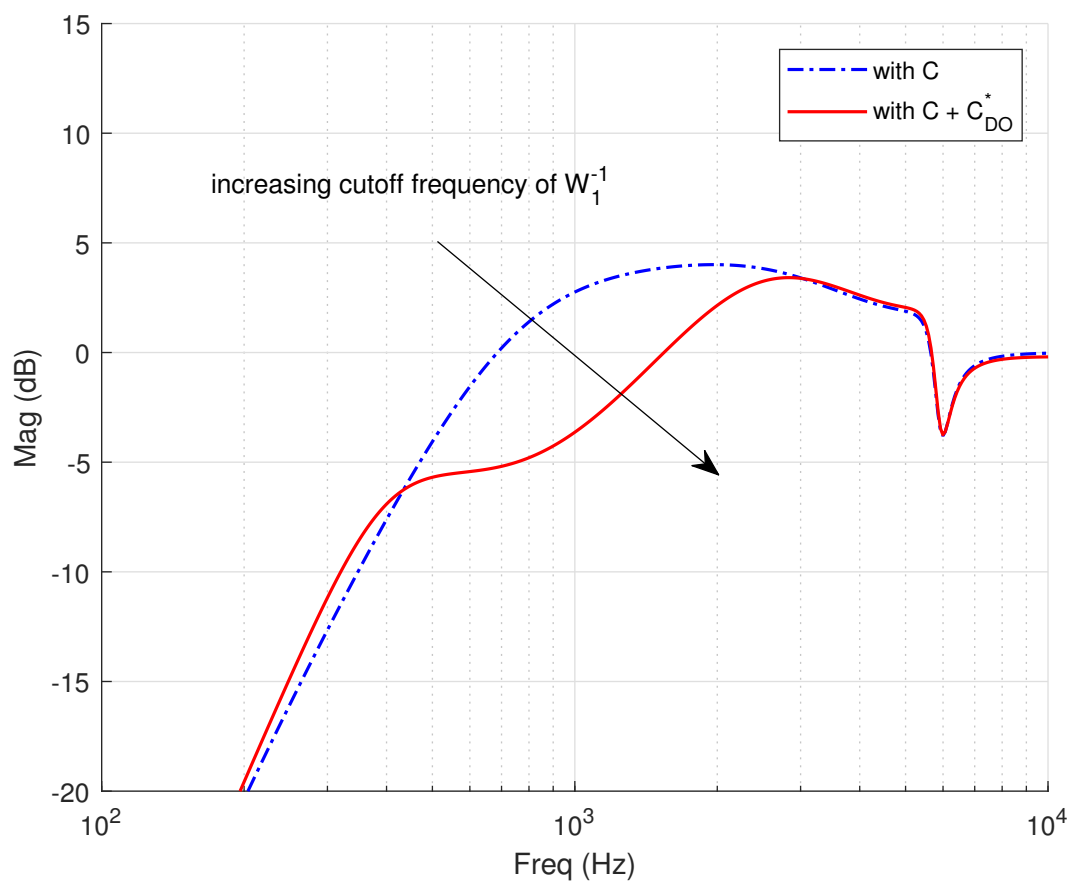

Figure 8. Compared sensitivity functions $\left(u_{P_{i n}} / d\right)$.

To evaluate time responses of the sensitivity functions, the following disturbances were introduced.

$$
\begin{aligned}
d(t) & =\sin (2 \pi 50 t)+0.5 \sin (2 \pi 500 t) \\
& +0.3 \sin (2 \pi 1000 t)
\end{aligned}
$$

The time responses to the disturbances are displayed in Figure 9. With the proposed DO, the disturbances are clearly reduced.

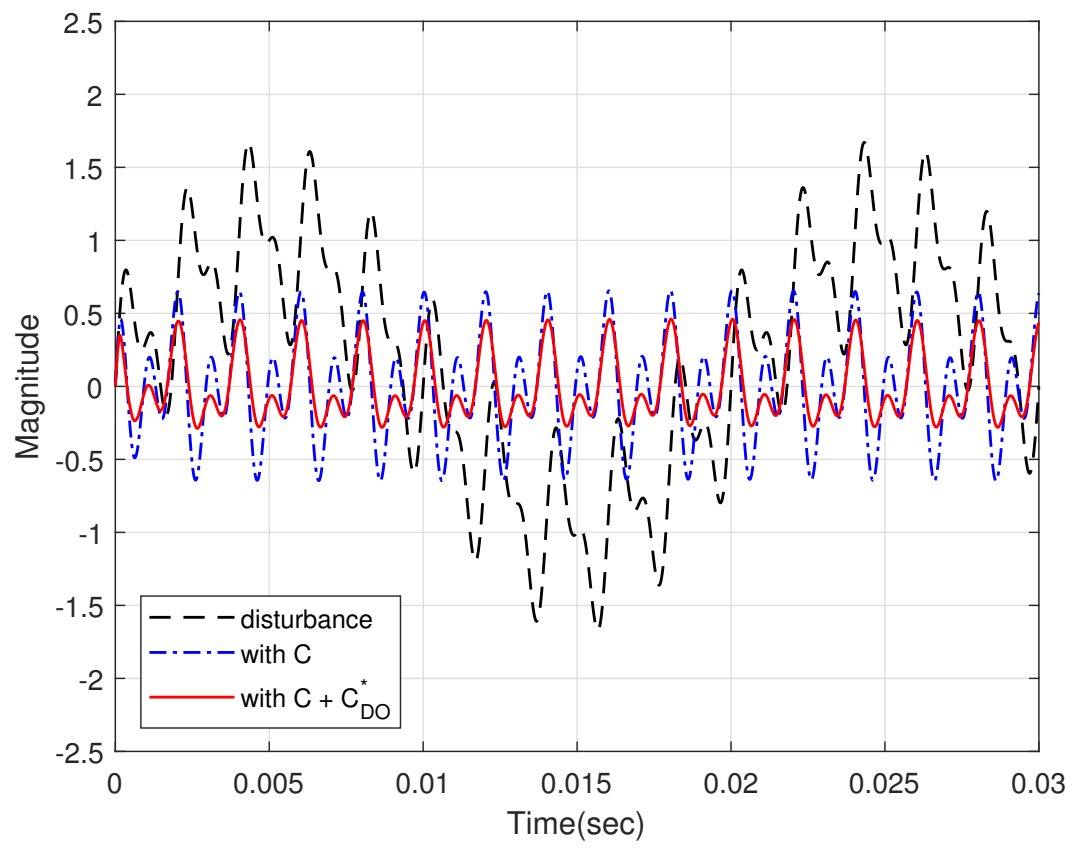

Figure 9. Time responses of sensitivity functions.

As the sensitivity function is improved, the torque transfer function is also improved as displayed in Figure 10. The torque disturbance function is defined by a transfer function from the introduced 
disturbances to the plant output. With the designed DO, the torque transfer function was also improved in the frequency range from $430 \mathrm{~Hz}$ to $3 \mathrm{kHz}$, whereas a small performance degradation could be observed at less than $430 \mathrm{~Hz}$.

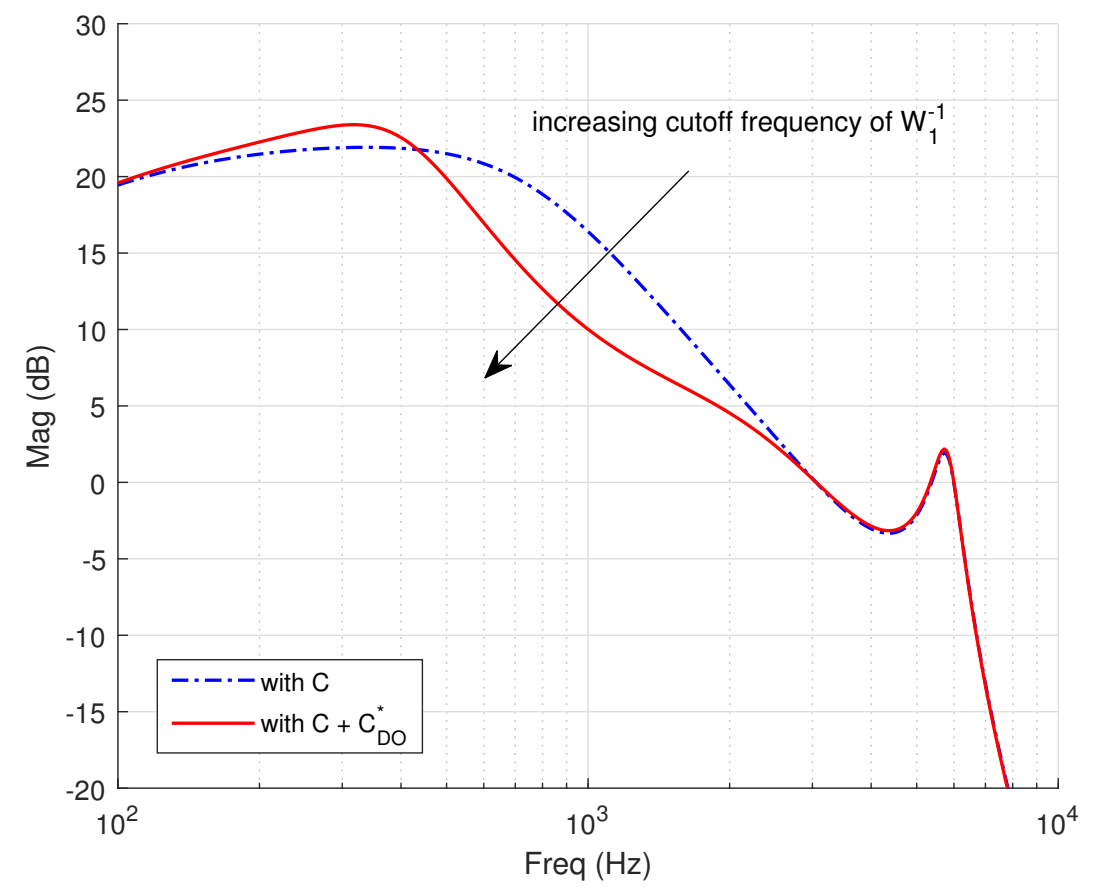

Figure 10. Torque transfer functions $(y / d)$.

Therefore, random disturbances were applied to fairly compare the performances. In Figure 11, the measured power spectral density and accumulated PES were compared.

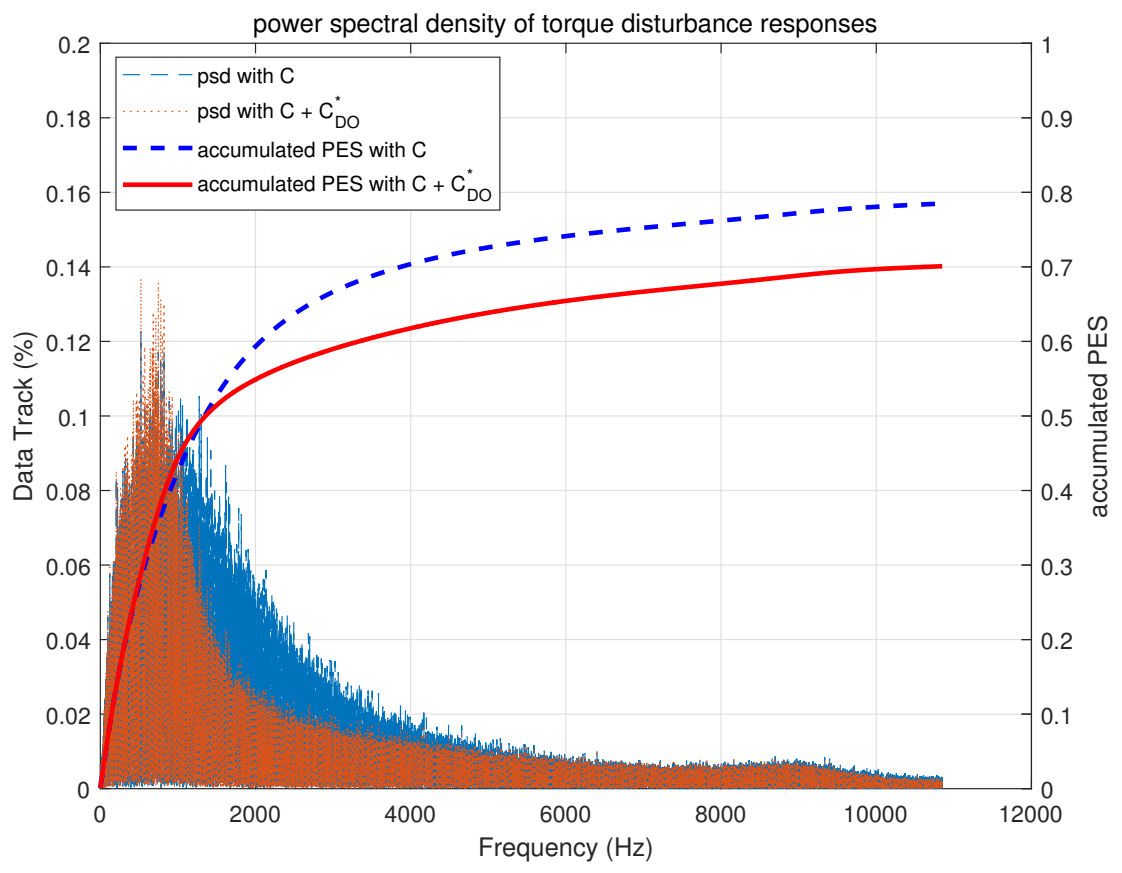

Figure 11. Power spectral densities and accumulated position error signal (PES).

With the proposed DO, the accumulated PES was improved by $10.8 \%$. 


\section{Discussion}

This section presents considerations and limitations of the proposed method. In addition, to relax the limitation of computation time, the required minimal controllers are summarized.

\subsection{Complete Solutions According to Model Types}

Equation (23) provides a complete solution of all types of models. However, to minimize the order of the controllers, minimally required controllers are summarized in Table 3 . In the table, the minimally required controller is presented to realize the DO; the optional filter could be used to reduce the high-frequency dynamics.

Table 3. Complete solutions based on model types.

\begin{tabular}{lll}
\hline Model Type & Minimally Required Controller & Optional Filter \\
\hline $\begin{array}{l}\text { stable or unstable, } \\
\text { minimum phase, } \\
\text { and bi-proper }\end{array}$ & $\frac{Q \cdot P_{n}^{-1}}{1-Q}$ & $\prod_{i=1}^{q} \frac{1}{s / \tau_{i}+1} \cdot \prod_{j=1}^{p} \frac{\tau_{j}^{2}}{s^{2}+2 \zeta_{j} \tau_{j} s+\tau_{j}^{2}}$ \\
\hline $\begin{array}{l}\text { stable or unstable, } \\
\text { minimum phase, } \\
\text { and strictly proper }\end{array}$ & $\frac{Q \cdot P_{n}^{-1}}{1-Q} \cdot \prod_{i=1}^{q} \frac{1}{s / \tau_{i}+1} \cdot \prod_{j=1}^{p} \frac{\tau_{j}^{2}}{s^{2}+2 \zeta_{j} \tau_{j} s+\tau_{j}^{2}}$ & - \\
\hline $\begin{array}{l}\text { stable or unstable, } \\
\text { non-minimum phase, } \\
\text { and bi-proper }\end{array}$ & $\frac{Q}{1-Q} \cdot \frac{A(s)}{B_{s}(s) \bar{B}(s)}$ & $\prod_{i=1}^{q} \frac{1}{s / \tau_{i}+1} \cdot \prod_{j=1}^{p} \frac{\tau_{j}^{2}}{s^{2}+2 \zeta_{j} \tau_{j} s+\tau_{j}^{2}}$ \\
\hline $\begin{array}{l}\text { stable or unstable, } \\
\text { non-minimum phase, } \\
\text { and strictly proper }\end{array}$ & $\frac{Q}{1-Q} \cdot \frac{A(s)}{B_{s}(s) \bar{B}(s)} \cdot \prod_{i=1}^{q} \frac{1}{s / \tau_{i}+1} \cdot \prod_{j=1}^{p} \frac{\tau_{j}^{2}}{s^{2}+2 \zeta_{j} \tau_{j} s+\tau_{j}^{2}}$ & - \\
\hline
\end{tabular}

Because the model discussed in Section 4 is stable, minimum phase, and strictly proper, the resulting solution was the second row of the solution table.

\subsection{Limitations and Considerations}

Let $O(\cdot)$ be the order of the corresponding transfer function. The order of the designed Q-filter is written as $O(Q)=O\left(W_{1}\right)+O\left(W_{2}\right)+O\left(P_{r}\right)$, and the minimal order of the DO is calculated by

$$
\begin{aligned}
O(D O) & =O(Q)+O\left(P_{n}\right) \\
& =O\left(W_{1}\right)+O\left(W_{2}\right)+O\left(P_{r}\right)+Q\left(P_{n}\right) \\
& =O\left(W_{1}\right)+O\left(W_{2}\right)+O\left(P_{i d}\right) .
\end{aligned}
$$

Accordingly, even though the proposed method stabilizes the DO, the resulting DO is a high-order controller. Compared conventional DOs, the order of the proposed controller is high. That is, in the proposed method, DO stability is achieved at the cost of complexity. In addition, the high-order controller delivers phase delays of the control efforts or incurs instability in the worst case scenario (when the required computational time is greater than the sampling time). Therefore, high-performance processors are required, or an effective computation method such as word length optimization in the digital implementation is necessary in the low-cost processor. If there is a margin on computation time, the optional filter as indicated in Table 3 can be used.

\section{Conclusions and Future Work}

This paper proposed a stability-guaranteed DO without destroying the existing DO structure. The authors preferred to maintain the DO structure because it has already been verified. To obtain the all-stabilizing filter, the LMI design was adopted. The two models were combined into a single model to be controlled to resolve the problems of adopting the DO design in the LMI frameworks. 
To solve the realization problem of the DO, two metrics were designed, and a single measurement was derived to quantify the two values simultaneously. The proposed method delivers complete solutions to all types of models. Application examples verified that the proposed method is valid, and the performance was indeed improved.

The proposed method offers guaranteed stability of a DO while maintaining a conventional DO structure. This is because the DO has a verified structure to effectively reduce external disturbances. Therefore, the proposed method requires a plant inverse model and a compensator to solve the causality problem, as in conventional DOs. Although the inverse model and the compensator are considered as a part of the Q-filter, the calculation can be a burden to control engineers. In the near future, the authors would prefer to remove the calculation while maintaining guaranteed stability. Thus, in future research, we expect that the conventional DO structure cannot be maintained.

Author Contributions: Conceptualization, S.S.; investigation, W.K. and S.S.; methodology, S.S.; writing-original draft preparation, S.S.; writing-review and editing, W.K.; funding acquisition, W.K. All authors have read and agreed to the published version of the manuscript.

Funding: This research was supported by Energy Cloud R\&D Program through the National Research Foundation of Korea(NRF) funded by the Ministry of Science, ICT (2019M3F2A1073313).

Acknowledgments: The author would like to appreciate the anonymous reviewers for their valuable comments and suggestions that have contributed to improve this manuscript.

Conflicts of Interest: The authors declare no conflict of interest.

\section{Abbreviations}

The following abbreviations are used in this manuscript:

$\begin{array}{ll}\text { DSA } & \text { dynamic signal analyzer } \\ \text { DO } & \text { Disturbance observer } \\ \text { GM } & \text { gain margin } \\ \text { LDV } & \text { laser Doppler vibrometer } \\ \text { PES } & \text { Position error signal } \\ \text { PM } & \text { Phase margin }\end{array}$

\section{References}

1. Ohnishi, K. A New Servo Method in Mechatronics. Trans. Jap. Soc. Electr. Eng. 1987, 107, 83-86.

2. Deng, J.; Ren, W.; Zhang, H.; Luo, Y.; Zhou, X.; Mao, Y. A Modified Disturbance Observer Structure Based on Acceleration Measurement for Disturbance Suppression in Tracking Control System. Appl. Sci. 2018, 8, 1571. [CrossRef]

3. Sariyildiz, E.; Ohnishi, K. Stability and Robustness of Disturbance-Observer-Based Motion Control Systems. IEEE Trans. Ind. Electron. 2015, 62, 414-422. [CrossRef]

4. Kim, W.; Chen, X.; Lee, Y.; Chung, C.C.; Tomizuka, M. Discrete-time Nonlinear Damping Backstepping Control with Observers for Rejection of Low and High Frequency Disturbances. Mech. Syst. Signal Proc. 2018, 104, 436-448. [CrossRef]

5. Kim, W.; Shin, D.; Won, D.; Chung, C.C. Disturbance Observer Based Position Tracking Controller in the Presence of Biased Sinusoidal Disturbance for Electro-hydraulic Actuators. IEEE Trans. Control Syst. Technol. 2013, 21, 2290-2298. [CrossRef]

6. Ba, D.X.; Dinh, T.Q.; Bae, J.; Ahn, K.K. An Effective Disturbance-Observer-Based Nonlinear Controller for a Pump-Controlled Hydraulic System. IEEE/ASME Trans. Mechatron. 2020, 25, 32-43. [CrossRef]

7. Huang, J.; Zhang, M.; Ri, S.; Xiong, C.; Li, Z.; Kang, Y. High-Order Disturbance-Observer-Based Sliding Mode Control for Mobile Wheeled Inverted Pendulum Systems. IEEE Trans. Ind. Electron. 2020, 67, 2030-2041. [CrossRef] 
8. Phadke, S.B.; Shendge, P.D.; Wanaskar, V.S. Control of Antilock Braking Systems Using Disturbance Observer With a Novel Nonlinear Sliding Surface. IEEE Trans. Ind. Electron. 2020, 67, 6815-6823. [CrossRef]

9. Guo, G.; Zhang, P. Asymptotic Stabilization of USVs with Actuator Dead-Zones and Yaw Constraints Based on Fixed-Time Disturbance Observer. IEEE Trans. Veh. Technol. 2020, 69, 302-316. [CrossRef]

10. Ahmed, N.; Chen, M.; Shao, S. Disturbance Observer Based Tracking Control of Quadrotor with High-Order Disturbances. IEEE Acess 2020, 8, 8300-8313. [CrossRef]

11. Suh, S. Unified $H_{\infty}$ Control to Suppress Vertices of Plant Input and Output Sensitivity. IEEE Trans. Control Syst. Technol. 2010, 18, 969-975. [CrossRef]

12. Park K.-S.; Suh, S. Discrete-time dual peak control for disturbance rejection. Microsyst. Technol. 2012, 18, 1645-1650. [CrossRef]

13. Xiao, D.; Nalakath, S.; Sun, Y.; Wiseman, J.; Emadi, A. Complex-Coefficient Adaptive Disturbance Observer for Position Estimation of IPMSMs With Robustness to DC Errors. IEEE Trans. Ind. Electron. 2020, 67, 5924-5935. [CrossRef]

14. Yun, J.N.; Su, J.-B. Design of a Disturbance Observer for a Two-Link Manipulator With Flexible Joints. IEEE Trans. Syst. Technol. 2014, 22, 809-815. [CrossRef]

15. Fan, C.; Xie, Z.; Liu, Y.; Li, C.; Liu, H. Adaptive Controller Based on Spatial Disturbance Observer in a Microgravity Environment. Sensors 2019, 19, 4759. [CrossRef]

16. Hong, Q.; Shi, Y.; Chen, Z. Adaptive Sliding Mode Control Based on Disturbance Observer for Placement Pressure Control System. Symmetry 2020, 12, 1057. [CrossRef]

17. Cao, P.; Gan, Y.; Dai, X. Finite-Time Disturbance Observer for Robotic Manipulators. Sensors 2019, $19,1943$. [CrossRef]

18. John, S.; Pedro, J.O. Neural network-based adaptive feedback linearization control of antilock braking system. Int. J. Artif. Intell. 2013, 10, 21-40.

19. Precup, R.-E.; Tomescu, M.L. Stable fuzzy logic control of a general class of chaotic systems. Neural Comput. Appl. 2015, 26, 541-550. [CrossRef]

20. Turnip, A.; Panggabean, J.H. Hybrid controller design based magneto-rheological damper lookup table for quarter car suspension. Int. J. Artif. Intell. 2020, 18, 193-206.

21. Garbouj, Y.; Dinh, T.N.; Raissi, T.; Zouari, T.; Ksouri, M. Optimal interval observer for switched Takagi-Sugeno systems: An application to interval fault estimation. IEEE Trans. Fuzzy Syst. 2020. [CrossRef]

22. Wang, L.; Su, J.; Xiang, G. Robust Motion Control System Design with Scheduled Disturbance Observer. IEEE Trans. Ind. Electron. 2016, 63, 6519-6529. [CrossRef]

23. Lyu, X.; Zhou, J.; Gu, H.; Li, Z.; Shen, S.; Zhang, F. Disturbance Observer Based Hovering Control of Quadrotor Tail-Sitter VTOL UAVs Using $H_{\infty}$ Synthesis. IEEE Robot. Autom. Lett. 2018, 3, 2910-2917. [CrossRef]

24. Lyu, X.; Zheng, M.; Zhang, F. $H_{\infty}$ Based Disturbance Observer Design for Non-minimum Phase Systems with Application to UAV Attitude Control. IEEE Xplore. 2018, [CrossRef]

25. Suh, S. Estimation error based disturbance observer design for flexible loop shaping. Electronics 2018, 7, 258. [CrossRef]

26. Suh, S. Discrete-time controller design to attenuate effects of external disturbances. Microsyst. Technol. 2009, 15, 1645-1651. [CrossRef]

27. Skogestad, S.; Postlethwaite, I. Multivariable Feedback Control: Analysis and Design; Wiley: New York, NY, USA, 2007; pp. 80-81.

28. Boyd, S.; Ghaoui, L.E.; Feron, F.; Balakrishnan, V. Linear Matrix Inequalities in System and Control Theory, 1994. Available online: https:/ / epubs.siam.org/doi/pdf/10.1137/1.9781611970777.bm (accessed on 15 May 2020).

29. Oliveira, C.D.; Geromel, J.C.; Bernussou, J. An LMI Optimization Approach to Multiobjective Controller Design for Discrete-Time Systems. In Proceedings of the 38th Conference on Decision and Control, Phoenix, AZ, USA, 7-10 December 1999; pp. 3611-3616. 
30. Skelton, E.; Iwasaki, T.; Grigoriadis, K. A Unified Algebraic Approach to Linear Control Design, 1997. Available online: https://www.researchgate.net/profile/Robert_Skelton3/publication/239666445_A_ Unied_Algebraic_Approach_to_Linear_Control_Design/links/545b86af0cf2f1dbcbcaf640/A-UniedAlgebraic-Approach-to-Linear-Control-Design.pdf (accessed on 15 May 2020).

31. Franklin, G.F.; Powell, J.D.; Abbas, E.-N. Feedback Control of Dynamic Systems; Prentice Hall: Upper Saddle River, NJ, USA, 2002; pp. 558-593

(C) 2020 by the authors. Licensee MDPI, Basel, Switzerland. This article is an open access article distributed under the terms and conditions of the Creative Commons Attribution (CC BY) license (http:/ / creativecommons.org/licenses/by/4.0/). 